\title{
La biométhylation des métaux chez l'homme et dans l'environnement : succès ou échec sur le plan toxicologique?
}

\section{The biomethylation of metals in man and in the environment : a success or a failure in toxicology?}

\section{Jean-Pierre ANGER ${ }^{(1) *}$, Laurence LABAT ${ }^{(2)}$, Michel LHERMITTE ${ }^{(2)}$}

(1) Université de Rennes 1, Faculté de Pharmacie, 2, avenue du Professeur Léon Bernard - 35043 RENNES Cedex Tél : 0299689239 -E-mail : jean-pierre.anger@wanadoo.fr (2) Université de Lille 2, Laboratoire de Toxicologie et Génopathies, Hôpital Calmette, Avenue du Pr. Jules Leclercq 59037 LILLE Cedex - Tél : 0320444951 - Fax : 0320444729 - E-mail : 1.labat@chru-lille.fr Tél : 0320444963 - Fax : 0320444729 - E-mail : mlhermitte@chru-lille.fr

*Auteur à qui adresser lạ correspondance : Jean-Pierre ANGER, 10, Allée de la Rivière - 35760 SAINT GRÉGOIRE

(Reçu le 23 juin 2005 ; accepté après modifications le 12 août 2005)

\section{RÉSUMÉ}

Le terme de biométhylation (méthylation biologique) correspond au transfert d'un groupement méthyle (-CH3), à partir d'un donneur de méthyle grâce à une enzyme, à l'intérieur d'un organisme vivant. Chez les procaryotes comme chez les mammifères, les donneurs biologiques sont le plus souvent la S-adénosylméthionine (S.A.M) et la méthylcobalamine (Vit B 12). Les enzymes qui contrôlent de tels transferts sont appelées méthyltransférases. Si la biométhylation de substances organiques sur les atomes de carbone, d'azote, de soufre ou d'oxygène est fréquente au cours du métabolisme chez tous les organismes supérieurs, d'autres éléments la subissent également : c'est le cas de plusieurs métaux et métalloïdes comme l'arsenic, le mercure, le bismuth ou l'étain. On sait aujourd'hui que ces composés méthylés présentent pour l'homme des risques non négligeables pour sa santé. Dans cette revue très générale et non exhaustive, nous rappelons la découverte et le mécanisme de biométhylation de quelques métaux et métalloïdes ayant entraîné au cours du siècle der-

\section{SUMMARY}

Biomethylation corresponds to the transfer of a methyl group $\left(-\mathrm{CH}_{3}\right)$ from a methyl donor to another compound by an enzymatic reaction. With germs as with mammals, biological donors are often the S-adenosylmethionin (SAM) and the methylcobalamin (Vit B12). Enzymes which control these transfers are called methyltransferases. If biomethylation of organic substances on carbon, nitrogen, sulphur or oxygen atoms is frequent during the metabolism with all the higher organisms, other elements are also concerned: it is the case of some metals and metalloids such as arsenic, mercury, bismuth or tin. We know today that these methylated compounds could be withpresent risks for human health. In this general and inexhaustive review, we recall the discovery and the biomethylation mechanism of some metals and metalloids responsible during the last century to dramatic human intoxications consecutively of an accidental contamination of environment (case of mercury in Minamata) or a therapeutic treatment (cases of bismuth and tin salts) or which appear 
nier; des intoxications dramatiques chez l'homme des suites d'une contamination accidentelle de l'environnement (cas du mercure à Minamata) ou d'une utilisation thérapeutique (cas des sels de bismuth et de l'étain) ou enfin qui apparaissent au cours du métabolisme de l'élément et dont on ignorait jusqu'alors la toxicité (cas de l'arsenic). Les circonstances et la symptomatologie de chaque intoxication sont succinctement évoquées.

Si la biométhylation des métaux dans l'environnement est en. fait un processus général de détoxication et donc un succès pour les microorganismes, il apparaît que la formation ou l'utilisation chez l'homme de ces composés organométalliques et organométalloïdiques. est plutôt un échec ainsi qu'en témoignent les intoxications gravissimes qui ont marqué l'histoire de la toxicologie au cours du vingtième siècle. L'imprudence, l'ignorance ou l'irresponsabilité en. ont été la cause. Il importe donc de surveiller la présence de ces formes organométalliques dans l'environnement et dans les milieux biologiques. Les méthodes modernes de spéciation alliant la séparation chromatographique (GC ou LC) à une détection très spécifique et très sensible comme l'ICP-MS permettent aujourd'hui de surveiller les niveaux d'exposition et devraient à l'avenir éviter de tels problèmes de santé.

\section{MOTS-CLÉS}

Biométhylation-métaux-arsenic-mercure-bismuth-étain.

\section{Introduction}

Le terme de biométhylation (méthylation biologique) correspond au transfert d'un groupement méthyle $\left(-\mathrm{CH}_{3}\right)$ à partir d'un donneur de méthyle grâce à une enzyme à l'intérieur d'un organisme vivant. Les enzymes qui contrôlent de tels transferts sont appelées méthyltransférases. Le terme plus général de transméthylation correspond à n'importe quelle réaction de transfert d'un groupement méthyle au sein de tout organisme vivant ou non. Ainsi la biométhylation équivaut à une transméthylation enzymatique se produisant dans les cellules.

La bioalkylation se réfère aux processus impliquant des groupements alkylés autres que le groupement méthyle, par exemple éthyle $\left(-\mathrm{C}_{2} \mathrm{H}_{5}\right)$ ou propyle $\left(-\mathrm{C}_{3} \mathrm{H}_{7}\right)$; ce processus est plus rare. On a cependant retrouvé dans certains gaz naturels des composés comme l'éthyldiméthylarsine As $\mathrm{C}_{2} \mathrm{H}_{5}\left(\mathrm{CH}_{3}\right)_{2}$ ou le triéthylarsine As $\left(\mathrm{C}_{2} \mathrm{H}_{5}\right)_{3}$ formés par action microbienne, le plus souvent ces formes alkyl-métal sont d'origine anthropogénique.

Si la biométhylation de substances organiques sur les atomes de carbone, d'azote, d'oxygène ou de soufre est fréquente au cours des réactions du métabolisme chez tous les organismes supérieurs, d'autres éléments la subissent également: c'est le cas de plusieurs métaux ou métalloïdes (As, Bi, Hg, Sn) dont on sait aujourd'hui que les dérivés méthylés présentent pour l'hom- during element metabolism with unknown toxicity till now (case of arsenic). Circumstances and symptomatology of each intoxication will be succinctly described. If metal biomethylation in environment is a general process of detoxification and a microbial success, it appears that human formation or use of those organometal or organometalloid compounds is rather a failure as it is shown with serious intoxication marked the toxicology of the $X X^{\text {th }}$ century.

Imprudence, ignorance or irresponsibility have been the cause. It is important to supervise these organometalic presences in environment and in biological samples. Actual methods of speciation with coupling chromatographic separation (GC or $L C$ ) and specific and sensible detection as $I C P-M S$ allow today surveillance of level expositions and should avoid these health problems.

\section{KEY-WORDS :}

Biomethylation-metals-arsenic-mercury-bismuth-tin.

me des risques toxicologiques non négligeables. En effet, dans la plupart des cas, la formation de tels composés augmente la biodisponibilité de l'élément qui peut ainsi s'accumuler le long des chaînes alimentaires. Tandis que les formes minérales de ces composés sont bien caractérisées (As, $\mathrm{Hg}$ ), et que certaines d'entre elles présentent une faible toxicité ( $\mathrm{Sn}, \mathrm{Bi}$ ), les composés méthylés formés, plus liposolubles peuvent se révéler particulièrement toxiques. Au cours du vingtième siècle l'homme a pu constater à ses dépens que la biométhylation ou plus généralement la bioalkylation des métaux pouvait avoir des conséquences désastreuses pour sa santé et l'environnement. L'intoxication par le méthylmercure (Maladie de Minamata), l'encéphalopathie bismuthique au cours du traitement des colopathies, la tentative malheureuse d'essayer de traiter la furonculose à l'aide d'un dérivé organique de l'étain (Affaire du Stalinon), plus récemment le risque de cancers lié à la présence d'arsenic dans l'eau de boisson et les considérables dégâts occasionnés chez les mollusques par le tributylétain en milieu aquatique ne sont là que quelques exemples qui auront permis de prendre conscience du danger de la bioalkylation que l'on considérait à tort autrefois, comme un mécanisme de détoxication.

Dans cette revue très générale, non exhaustive, nous rappelons la découverte et les mécanismes de biométhylation de quelques métaux et métalloïdes : arsenic, mercure, étain, bismuth, ayant entraîné des intoxica- 
tions dramatiques chez l'homme des suites d'une contamination accidentelle de l'environnement ou d'une utilisation thérapeutique et qui ont jalonné l'histoire de la toxicologie au cours du siècle dernier. Ces catastrophes devraient être plus facilement évitées aujourd'hui grâce aux nouvelles approches expérimentales in vitro de la toxicologie moléculaire et surtout aux immenses progrès de l'analyse toxicologique par l'apport des méthodes de spéciation.

\section{Considérations générales sur la biométhylation}

La biométhylation- est un processus métabolique important, largement distribué chez tous les organismes vivants des systèmes géochimiques et anthropogéniques (1). Plusieurs substances naturelles méthylées sur un atome d'azote ont été découvertes au début du $19^{\text {smo }}$ siècle : la créatine, la créatinine, la choline, la triméthylamine ainsi que, la triméthylglycine ou bétaïne. On doit la première observation de méthylation biologique à His (2) qui, s'intéressant à la détoxication des composés aromatiques, administra de la pyridine à un chien et constata la présence de $\mathrm{N}$-méthylpyridine dans ses urines. D'autres exemples de biométhylation furent démontrés ultérieurement : la conversion de la xanthine en méthylxanthine chez le lapin ou celle de l'acide nicotinique en trigonelline chez le chien (3). Par la suite divers travaux ont montré que les rats pouvaient substituer l'homocystéine par la méthionine dans leur régime en présence de choline, conduisant au concept de transméthylation (transfert d'un groupement méthyle d'un composé à un autre pour former des composés $\mathrm{N}-\mathrm{CH}_{3}$ ou S- $\mathrm{CH}_{3}$ ). Le rôle très important de la méthio- nine composé S-méthylé fut lentement reconnu, le groupement méthyle ne pouvant être transféré qu'à partir d'un composé riche en énergie, la S-adénosylméthionine (4). Le rôle de la S-adénosylméthionine, donneur de méthyle dans des centaines de réactions de méthylation est maintenant bien établi. Le mécanisme de la biosynthèse «de novo » de groupements méthyles a également été déterminé. Du fait de sa large distribution et de ses nombreuses utilisations en agriculture, dans l'industrie ou même en thérapeutique, la plupart des recherches concernent l'arsenic. Comme l'azote, l'arsenic est membre du groupe 15 dans la classification périodique des éléments, au même titre d'ailleurs que l'antimoine et le bismuth. Pour l'arsenic, les principaux composés volatils formés par méthylation possèdent la structure $\left.\left(\mathrm{CH}_{3}\right)_{\mathrm{n}} \mathrm{As} \mathrm{H}{ }_{3-\mathrm{n}}\right)$ où $\mathrm{n}=1,2$ ou 3 ; ce sont respectivement les mono-, di- et triméthylarsine (MMA, DMA et TMA). Les principaux composés non volatils sont le monométhylarsonate $\left(\mathrm{MMAs}^{\mathrm{v}}\right)$, le diméthylarsonate (DMAs ${ }^{v}$ ) et le triméthylarsonate $\left(\right.$ TMAs $^{v}$ ) (Tableau I).

\section{Historique de la méthylation de l'arsenic}

Les efforts conjugués des mycologues, des microbiologistes, des chimistes, des biochimistes et plus récemment des spécialistes en biologie moléculaire ont contribué à mieux comprendre les mécanismes de la biométhylation de l'arsenic. Dans une série de travaux entrepris au début des années 30 , Fréderick Challenger et son équipe (5) apportèrent la preuve chimique que des produits mono-, di- et triméthylés pouvaient être formés par les microorganismes exposés à de l'arsenic inorganique (arsénite) . En 1945, Challenger publia une revue sur la méthylation biologique et proposa même un mécanisme

Tableau I: Principaux composés arséniés dans l'environnement.

\begin{tabular}{|l|l|l|}
\hline Composés & Formule & Valence \\
\hline Arsine & $\mathrm{AsH}$ & -3 \\
\hline Monométhylarsine (MMA) & $\mathrm{CH}_{3} \mathrm{AsH}_{2}$ & -3 \\
\hline Diméthylarsine (DMA) & $\left(\mathrm{CH}_{3}\right) 2 \mathrm{AsH}$ & -3 \\
\hline Triméthylarsine (TMA) & $\left(\mathrm{CH}_{3}\right)_{3} \mathrm{As}$ & -3 \\
\hline Acide arsénieux & $\mathrm{As}(\mathrm{OH})_{3}$ & +3 \\
\hline Acide arsénique & $\mathrm{AsO}(\mathrm{OH})_{3}$ & +5 \\
\hline Acide monométhylarsonique (MMAs & ) & +5 \\
\hline Acide diméthylarsonique (DMAs $)$ ou Acide cacodylique & $\mathrm{CH}_{3} \mathrm{AsO}(\mathrm{OH})_{2}$ & $\left(\mathrm{CH}_{3}\right)_{2} \mathrm{AsO}(\mathrm{OH})$ \\
\hline Oxyde de triméthylarsine (TMAO) & $\left(\mathrm{CH}_{3}\right)_{3} \mathrm{AsO}$ & +5 \\
\hline Arsénobétä̈ne & $\left(\mathrm{CH}_{3}\right)_{3} \mathrm{As}^{+} \mathrm{CH}_{2} \mathrm{COO}^{-}$ & +5 \\
\hline Arsénocholine & $\left(\mathrm{CH}_{3}\right)_{3} \mathrm{As}^{+} \mathrm{CH}_{2} \mathrm{CH}_{2} \mathrm{OH}$ & +5 \\
\hline Arsénosucres & & +5 \\
\hline
\end{tabular}


pour expliquer la biométhylation de l'arsenic :

$$
\begin{aligned}
& \mathrm{As}^{\mathrm{III}} \mathrm{O}_{3}^{--}+\mathrm{CH}_{3}{ }^{+} \rightarrow \mathrm{CH}_{3} \mathrm{As}^{\mathrm{v}} \mathrm{O}_{3}^{-}+2 \mathrm{e} \rightarrow \mathrm{CH}_{3} \mathrm{As}^{\mathrm{III}} \mathrm{O}_{2}^{-}+\mathrm{CH}_{3}{ }^{+} \\
& \rightarrow\left(\mathrm{CH}_{3}\right)_{2} \mathrm{As}^{\vee} \mathrm{O}_{2}^{-}+2 \mathrm{e}^{-} \rightarrow\left(\mathrm{CH}_{3}\right)_{2} \mathrm{As}^{\mathrm{III}} \mathrm{O}^{-}+\mathrm{CH}_{3}{ }^{+} \\
& \rightarrow\left(\mathrm{CH}_{3}\right)_{3} \mathrm{As}^{v} \mathrm{O}
\end{aligned}
$$

Ce schèma où la méthylation oxydative de As alterne avec la réduction de $A s^{\mathrm{V}}$ en $\mathrm{As} \mathrm{s}^{\mathrm{II}}$ suggérait immédiatement deux questions :

1) Quelle est la source des groupements méthyles nécessaires à la formation des méthylarsonates?

\section{2) Comment $A s^{v}$ est-il réduit en As ${ }^{\text {III }}$ ?}

Challenger (6) suggéra comme réponse à la première question le rôle du formaldéhyde comme source de groupements méthyles. L'identification de la S-adénosylméthionine (SAM), donneur presque universel de groupements $\mathrm{CH}_{3}$ chez les mammifères et la mise en évidence de nombreuses méthyltransfèrases SAM dépendantes ont définitivement répondu à la première question. La seconde question concernant l'origine du pouvoir réducteur qui convertit $\mathrm{As}^{\mathrm{V}}$ en $\mathrm{A} \mathrm{s}^{\mathrm{III}}$ comme prérequis à sa méthylation est plus difficile à comprendre et demeure toujours un objectif de la recherche (7).

\section{Mécanismes de la biométhylation de l'arsenic}

La biométhylation de l'arsenic est très répandue : elle se produit non seulement chez les microorganismes mais également chez les algues, les plantes et les mammifères.

\section{Chez les champignons et les levures}

La libération d'arsenic par les champignons a été décrite pour la première fois, en 1893. Le produit qui a une odeur caractéristique d'ail, est parfois connu comme le gaz Gosio (8). L'addition d'oxydes arséniés, de MMA ou de DMA dans les cultures de champignons Penicillium brevicaule entraîne la production de TMA. $\mathrm{La}$ réduction de l'arséniate en TMA serait la suivante :

$$
\begin{aligned}
& \mathrm{AsO}_{4}-\mathrm{AsO}_{3}{ }^{-} \rightarrow\left(\mathrm{CH}_{3}\right) \mathrm{AsO}_{3}{ }^{-} \rightarrow\left(\mathrm{CH}_{3}\right)_{2} \mathrm{AsO}_{2}{ }^{-} \\
& \rightarrow\left(\mathrm{CH}_{3}\right)_{3} \mathrm{As}
\end{aligned}
$$

Les groupements thiols $(-\mathrm{SH})$ endogènes jouent un rôle primordial dans la transformation des espèces arséniées inorganiques (Fig 1). Le glutathion (G-SH) agit comme un agent réducteur de $A s^{\mathrm{v}}$ et l'As $s^{\mathrm{II}}$ produit, peut alors accepter un groupement méthyle du donneur SAM pour former de l'arsenic méthylé (9). Les produits finaux vont dépendre de l'organisme responsable de cette réaction. Par exemple, dans le cas des champignons, le produit final de la réaction sera l'oxyde triméthylarsine (TMAO) ou le triméthylarsine (TMA).

Des études plus récentes ont montré que les espèces fongiques telles que Candida humicola, Gliocladium roseum et Penicillium sp sont capables de transformer l'acide méthylarsonique en DMA, lui même transformé en TMA. Cox et Alexander (1973), cités par Laperche (10) ont montré que la levure Candida humicola pouvait méthyler l'arséniate, l'arsénite, le méthylarsonate, le diméthylarsonate et l'oxyde triméthylarsine en TMA.

\section{Chez les bactéries}

La méthylation bactérienne de l'arsenic inorganique a été très étudiée chez les bactéries méthanogènes, en condition anaérobie. Ces bactéries vont produire du méthane comme produit final de leur métabolisme. La voie de production de diméthylarsine par Methanobacterium est représentée sur la figure 1. L'arséniate est premièrement réduit en arsénite, ensuite méthyłé, via le donneur de groupement méthyle (méthylcobalamine), pour former l'acide méthylarsonique. Ce dernier composé est réduit et méthylé pour former l'acide diméthylarsinique qui sera lui même réduit en diméthylarsine. Il faut noter que la réaction de méthylation de l'acide méthylarsonique en acide diméthylarsinique est inhibée par un excès d'arsénite. La méthylation de l'arsenic organique est couplée à la biosynthèse du méthane et se trouve être un des mécanismes de détoxification de l'arsenic (11).

En condition aérobie, des bactéries isolées de l'environnement (Proteus sp., Escherichia coli et Flavobacterium sp., Corynebacterium sp., Pseudomonas sp.) préacclimatées à des concentrations de $100 \mathrm{mg} / \mathrm{l}$ d'arséniate de sodium sont capables de le réduire en arsénite puis de le méthyler en méthylarsines volatils. Les bactéries Serratia marinorubra, Aeromonas sp., Escherichia coli, et Flavobacterium sont capables de transformer l'arsenic accumulé dans leurs cellules en formes arseniées mono- et diméthylées. La transformation de l'arsenic en espèces triméthylées serait plutôt le fait des algues marines et d'eaux douces. La biométhylation dans l'environnement est ainsi un mécanisme de résistance développé par les microorganismes car les composés arseniés méthylés sont beaucoup moins toxiques que les formes d'arsenic inorganiques. Différents microorganismes bactériens et fongiques sont capables de méthyler l'arsenic inorganique présent dans le sol. La voie de méthylation des bactéries et des champignons est différente. La biométhylation par les bactéries aura comme produit final le diméthylarsine ou le triméthylarsine. Par contre les champignons sont capables de transformer les composés arséniés inorganiques et organiques en arsonates méthylés non volatils. Notons qu'il se produit également dans les sols le processus inverse qui est une déméthylation des. composés organoarséniés. Ces composés sont minéralisés pour former de l'arsenic inorganique et du gaz carbonique (10). 
A) Chez les champignons et les levures : Candida humicola, Penicillium
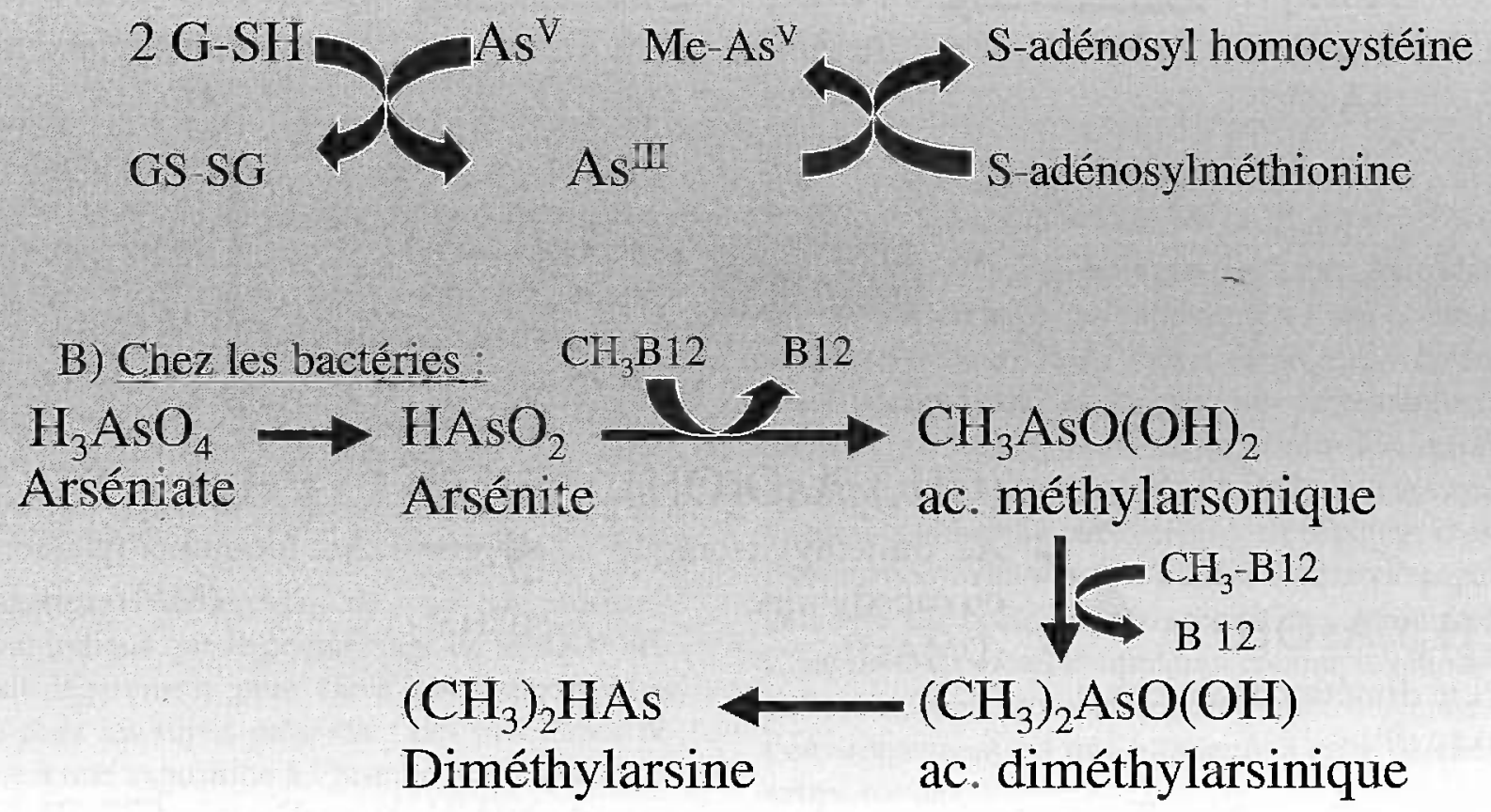

Figure 1 : Méthylation de l'arsenic.

\section{Méthylation de l'arsenic chez l'Homme}

Chez de nombreux mammifères y compris l'Homme, l'arsenic inorganique (iAs) est réduit dans le sang de sa forme pentavalente (arséniate) en arsenic trivalent (arsénite), capté par les hépatocytes, où il subit une méthylation oxydative le transformant successivement en acide monométhylarsonique (MMAs ${ }^{v}$ ) puis en acide diméthylarsonique (DMAsv) ou acide cacodylique, éliminés par l'urine. Cette biotransformation se déroule donc en deux étapes successives (Fig 2) :

- une étape de réduction assurée par le glutathion (G-SH) en présence d'une $A s^{v}$ réductase ;

- une étape de méthylation oxydative catalysée par une $\mathrm{As}^{\mathrm{III}}$ méthyltransférase appelée CYT 19 par Styblo et al. (12), qui utilise la S-adénosylméthionine (SAM) comme donneur de méthyle (13). La SAM est alors convertie en S-adénosylhomocystéine ( $\mathrm{SAH}$ ) dont la régénération en SAM est possible grâce au 5-10 méthyltétrahydrofolate (THF), à la vitamine $\mathrm{B} 6$ ou à la vitamine B12 (14).

Les méthyltransférases impliquées dans la méthylation de l'arsenic n'ont pas encore été toutes caractérisées. Des enzymes ont été purifiées à partir de foie de lapin, de hamster et de singe Rhésus et partiellement caractérisées $(15,16)$. L'arsénite méthyltransférase et la MMA méthyltransférase semblent appartenir à des protéines de poids moléculaire voisin de $60 \mathrm{kDa}$, chacune ayant ses propres caractéristiques. Une activité méthyltransférase a également été detectée dans des cultures d'hépatocytes humains (17).

Des études expérimentales montrent que le foie est le site le plus important de méthylation de l'arsenic, spécialement après ingestion (18). Cependant As peut être méthylé dans d'autres tissus (testicules, rein et poumon), chez la Souris mâle (19).

\section{Facteurs modifiant la méthylation de As chez} l'Homme

Puisque le MMAs ${ }^{v}$ et le DMAs ${ }^{v}$ sont rapidement excrétés dans l'urine, l'évaluation de la méthylation de As est principalement basée sur les taux relatifs de ces deux métabolites urinaires. Le calcul montre qu'il existe de très grandes variations dans le métabolisme de iAs entre les mammifères. D'ailleurs les études sur les As méthyltransférases hépatiques de divers animaux apportent la preuve d'une diversité marquée selon les espèces (19). Contrairement à la plupart des autres mammifêres, l'Homme excrète des quantités appréciables de MMAs ${ }^{\mathrm{v}}$ dans l'urine (20). La Souris et le Chien sont également très efficaces à méthyler l'arsenic en DMAs ${ }^{v}$ ce qui se traduit par une rapide excrétion urinaire de ce métabolite et une faible rétention de l'arsenic dans les tissus. Les Rats aussi méthylent bien l'arsenic inorganique mais une grande partie du diméthylarsonate formé est retenu dans les érythrocytes et il 


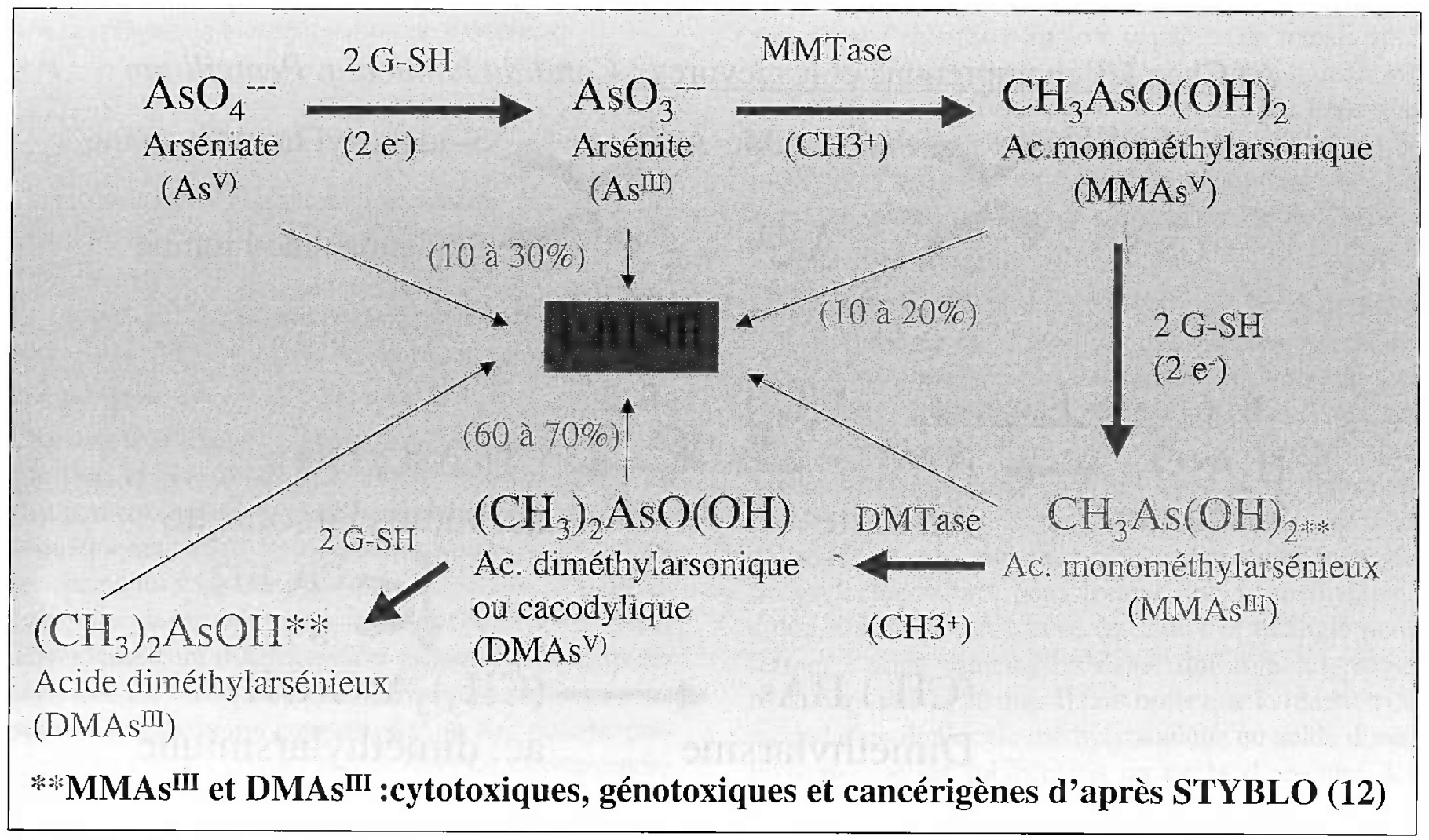

Figure 2 : Méthylation de As chez l'homme.

en résulte une faible excrétion urinaire. Par contre le Singe et le Cobaye n'ont pas la possibilité de méthyler l'arsenic inorganique(20).

Chez l'Homme, il existe également des variations dans la méthylation de As entre différents groupes de population. Quelque soit le type et la nature de l'exposition, la distribution relative moyenne des métabolites de l'arsenic dans l'urine semble être assez constante :10$30 \%$ de As inorganique, $10-20 \%$ de $\mathrm{MMAs}^{\mathrm{v}}$ et $60-70 \%$ de DMAsv (20). Cependant il y a des exceptions. Certaines peuplades indigènes des Andes, vivant dans le nord du Chili et de l'Argentine où l'eau du sol est fortement contaminée par l'arsenic, excrètent moins de $M M A s^{v}$ dans l'urine, souvent un très faible pourcentage (19). Par ailleurs, des personnes vivant dans certaines régions de Taiwan semblent avoir un pourcentage élevé inhabituel (20-30\% en moyenne) de MMAs ${ }^{v}$ dans l'urine $(21,22)$. Ces données semblent indiquer la possibilité d'un polymorphisme génétique dans la régulation des méthyltransférases impliquées dans la méthylation de l'arsenic (21).

Enfin il existe des variations inter individuelles notables dans le métabolisme de l'arsenic (17). Des études expérimentales ont montré que divers facteurs : niveau de dose, voie d'administration, nature de l'arsenic administré, état nutritionnel, etc..., peuvent moduler la méthylation de l'arsenic inorganique. Cependant les facteurs de régulation se rapportant à la méthylation de l'arsenic chez l'Homme sont peu connus. Quelques travaux indiquent que l'âge (23) et la grossesse (24) pourraient jouer un rôle significatif.

\section{Méthylation de l'arsenic et toxicité}

Les produits de méthylation de l'arsenic inorganique (iAs), le $\mathrm{MMAs}^{\mathrm{v}}$ et le DMAsv sont nettement moins réactifs avec les constituants cellulaires et plus rapidement excrétés dans l'urine que l'arsenic inorganique (25). D'ailleurs lorsque l'on compare les toxicités aiguës expérimentales, on note effectivement que le $\mathrm{MMAs}^{\mathrm{v}}$ et le DMAs ${ }^{\mathrm{v}}$ sont moins toxiques que l'arsenic minéral trivalent ou pentavalent, chez le rongeur (Tableau II). En ce sens la méthylation de l'arsenic a longtemps été considérée comme un processus de détoxication. Cependant des travaux récents ont rapporté que le MMAs ${ }^{\text {III }}$ et/ou le DMAs $s^{\text {III }}$ formés au cours du métabolisme de l'arsenic et mis en évidence dans l'urine d'individus chroniquement exposés à l'arsenic minéral (26) ainsi que dans des cultures d'hépatocytes humains exposés à différentes concentrations de iAs ${ }^{\text {III }}$ (27), se sont révélés des inhibiteurs enzymatiques cellulaires plus puissants que iAs ${ }^{\mathrm{v}}$ ou iAs ${ }^{\mathrm{II}}$. Dans les essais de viabilité cellulaire au cours d'études in vitro, le

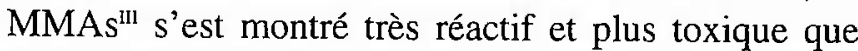
$A s^{\text {III }}$ ou $A s^{v}$ vis à vis des hépatocytes humains, des kératinocytes et des cellules épithéliales bronchiques 
(11). D'autres études in vitro démontrent que MMAs ${ }^{\text {III }}$ et DMAs ${ }^{\text {III }}$ sont génotoxiques et beaucoup plus puissants à altérer l'ADN que l'iAs $s^{\text {ill }}$ (28). Il apparaît donc essentiel de comprendre aujourd'hui la contribution importante de ces deux métabolites intermédiaires dans la toxicité de l'arsenic. Diverses hypothèses ont d'ailleurs été proposées pour expliquer le pouvoir cancérogène de l'arsenic mais les mécanismes n'en sont pas totalement élucidés. Il semblerait que As n'agit pas selon des mécanismes génotoxiques et mutagéniques classiques mais sans doute comme un promoteur de tumeur qui modifierait les signaux de transduction impliqués dans la croissance et la prolifération cellulaire (28). La biométhylation est donc bien ici un processus qui favorise la toxicité et la cancérogénicité de l'arsenic.

Il est également très intéressant de noter que plusieurs travaux ont indiqué que le pourcentage de MMAs ${ }^{\mathrm{v}}$ urinaire était légèrement plus élevé par rapport à la moyenne chez les sujets présentant des problèmes de santé suite à une exposition à l'arsenic. Cette constatation serait-elle due à une rétention du MMAs ${ }^{\mathrm{II}}$ dans les tissus ainsi que le suggère Vahter ? (20). Au Mexique, certaines personnes présentent des effets cutanés suite à la consommation d'eau contaminée par l'arsenic : elles ont un taux urinaire de MMAsv plus élevé, de l'ordre de $5 \%$ et un taux de DMAs ${ }^{v}$ plus faible par rapport à la population non touchée (29). De même, à Taiwan, les personnes sujettes à lésions cutanées arsenicales ont un taux de iAs et de MMAsv plus élevé et un taux de DMAs ${ }^{\mathrm{v}}$ plus faible par rapport à la population témoin (30). Le nombre des aberrations chromosomiques structurelles dans les lymphocytes périphériques de populations finlandaises exposées à l'arsenic présent dans l'eau de boisson est également associé à un taux de MMAs ${ }^{v}$ urinaire croissant alors que le taux de DMAs ${ }^{v}$ est diminué (31). D'autres études sur le rôle des métabolites méthylés dans la toxicité de l'arsenic sont, bien entendu nécessaires, mais si on retrouve un taux significatif de MMAs ${ }^{\text {III }}$ dans les tissus à la suite

Tableau II : Toxicité aiguë comparée de quelques composés arséniés.

\begin{tabular}{|l|c|c|c|}
\hline Composé & Ditsomg kg & Rspece & Voie d administration \\
\hline $\mathrm{As}_{2} \mathrm{O}_{3}$ & 34,5 & Souris & orale \\
$\mathrm{Na}_{2} \mathrm{AsO}_{3}$ & 4,5 & Rat & intra-péritonéale \\
$\mathrm{Na}_{3} \mathrm{AsO}_{4}$ & $14-18$ & Rat & intra-péritonéale \\
$\mathrm{MMAsV}$ & 1800 & Souris & orale \\
DMAsV & 1200 & Souris & orale \\
Arsénobétaïne & 10000 & Souris & orale \\
Arsénocholine & 6500 & Souris & orale \\
TMAO & 10600 & Souris & orale \\
TMA & 8000 & Souris & sous-cutané \\
\hline
\end{tabular}

d'une exposition à l'arsenic inorganique, on devrait s'attendre à ce que les gens qui éliminent davantage de $\mathrm{MMAs}^{\mathrm{v}}$ retiennent plus de MMAs ${ }^{\mathrm{III}}$ dans leur organisme que ceux qui présentent un taux urinaire normal de ce métabolite.

\section{Cas du mercure}

Le mercure, élément chimique de numéro atomique 80 , est le seul métal liquide à la température ordinaire. Il tire son symbole $\mathrm{Hg}$ du mot grec latinisé « hydrargyrum » (argent liquide). Le mercure présente un risque majeur pour l'écosystème marin et pour le consommateur humain, en raison de sa toxicité et de ses capacités de biomagnification par la chaîne alimentaire. Il est utilisé dans des activités très variées (industrie papetière, industrie du bois, industrie chimique, agriculture...). C'est donc un métal ubiquitaire, comme le plomb ou le cadmium, mais dont la concentration moyenne dans l'environnement est très inférieure à celle de ces deux autres métaux.

\section{Le cycle biogéochimique du mercure}

Le cycle global du mercure est dominé par les échanges entre le milieu aquatique et l'atmosphère. En milieu aqueux, deux réactions chimiques essentielles sont en compétition : la réduction et la méthylation. La première favorise le recyclage atmosphérique, la seconde la bioaccumulation. Le mercure est émis dans l'atmosphère sous forme de $\mathrm{Hg}^{0}$ gazeux et de $\mathrm{Hg}^{2+}$ gazeux et particulaire. Les sources naturelles sont constituées par les sols et les roches mercurifères, le volcanisme et la biomasse, en particulier via les feux de forêt. Les émissions anthropogéniques directes ou indirectes, les réemissions proviennent aussi surtout des processus de combustion. Les $70 \%$ de la surface de la planète couverts d'eau sont aussi source de mercure pour l'atmosphère. Les eaux de surface abritent la formation de $\mathrm{Hg}^{0}$ volatil par photoréduction et réduction enzymatique du $\mathrm{Hg}^{2+}$. Les océans et mers côtières émettent dans l'atmosphère du mercure essentiellement sous forme élémentaire $\mathrm{Hg}^{0}$. La présence de mercure dans l'eau de pluie qui retombe sur les continents et la mer résulte à la fois de la solubilité de $\mathrm{Hg}^{0}$ dans l'eau, de son oxydation et de son adsorption subséquente sur les aérosols. La forme monométhylée ( $\mathrm{MeHg}$ ou $\mathrm{CH}_{3} \mathrm{Hg}^{+}$), issue de la décomposition du diméthylmercure $\left(\mathrm{CH}_{3}\right)_{2} \mathrm{Hg}$, constitue moins de $1 \%$ du mercure dans les précipitations. Le séjour du $\mathrm{Hg}$ dans l'atmosphère sous forme de particules est très court, de l'ordre de quelques jours. Par contre, en phase vapeur, il est de l'ordre d'une année. 


\section{Méthylation du mercure}

Dans l'environnement, le mercure se transforme en méthylmercure par l'ajout d'un ou deux groupement(s) méthyle $\left(\mathrm{CH}_{3}\right)$. La méthylation de $\mathrm{Hg}^{2+}$ est un processus biologique naturel très commun sous l'action de la méthylcobalamine, produite par des bactéries stagnartes du milieu marin. Cette transformation entraîne la production de composés de méthylmercure, fortement toxiques ainsi que leur bioaccumulation dans les tissus vivants avec augmentation de leur concentration tout au long de la chaîne alimentaire, depuis les microorganismes comme le plancton, aux petits poissons, puis à des espèces qui s'en nourrissent comme les loutres, les chats et chiens, et au bout de la chaîne, les humains.

On pense qu'une grande variété de micro-organismes, principalement les bactéries sulfato-réductrices, ainsi que les bactéries méthanogènes (qui produisent du méthane) participent à cette conversion $\mathrm{de} \mathrm{Hg}^{2+}$ en $\mathrm{MeHg}$ dans certaines conditions anaérobiques que l'on trouve, par exemple, dans les zones humides et les sédiments de rivière, ainsi que dans certains types de sol. La méthylation s'effectue principalement dans l'environnement aquatique à $\mathrm{pH}$ acide avec des concentrations élevées de matière organique ; c'est ce qui s'est passé à Minamata. Les taux de biométhylation sont liés à des variables écologiques qui ont une incidence sur la disponibilité des ions mercuriques ainsi qu'à la taille des populations des microbes assurant la méthylation. Le processus augmente avec des températures plus chaudes qui favorisent une productivité biologique, et diminue pendant l'hiver.

\section{Métabolisme et toxicité du mercure chez l'homme}

Chez l'Homme, le mercure élémentaire ou métallique $\mathrm{Hg}^{0}$ est transporté dans les différents organes où il est oxydé en ion mercurique $\mathrm{Hg}^{2+}$ qui peut se lier aux protéines sanguines et tissulaires. Le méthylmercure subit dans les tissus une biotransformation en composés mercuriques divalents par rupture de la liaison carbonemétal. À la différence de l'arsenic, il ne semble pas que le mercure puisse être méthylé dans les tissus, chez les mammifères (32).

L'intoxication chronique par le mercure minéral, initialement décrite sous le nom d'hydragyrisme, peut s'observer à la suite d'une exposition professionnelle aux vapeurs de mercure et se caractérise par une triade symptomatique particulière : stomatite avec liseré gingival, néphrite chronique et neurotoxicité se traduisant par des tremblements intentionnels. Cette neurotoxicité se retrouve également, de façon plus insidieuse, dans la maladie de Minamata (33).

\section{La maladie de Minamata}

Dans les années 50, une épidémie mystérieuse se déclencha dans la baie de Minamata, au sud du Japon. Les troubles affectaient plus particulièrement les familles de pêcheurs (malformation des nouveau-nés, dysfonctionnement du système nerveux) et les animaux domestiques (chats atteints de convulsions, se jetant dans la mer du haut des falaises), tous deux gros consommateurs de poissons.

Les analyses montrèrent qu'une usine de produits chimiques fabriquant du plastique (PVC - chlorure de polyvinyle) utilisait le mercure comme catalyseur qui était rejeté en mer après usage. Bien que le mercure soit sous forme inorganique (oxyde de mercure), peu biodisponible et peu toxique, la condensation, la salinité et l'activité bactérienne des micro-organismes marins transformèrent le mercure en une forme organique (méthylmercure), très biodisponible et très toxique.

Des analyses de vase révélèrent des concentrations de mercure dans les sédiments pouvant atteindre plus de deux grammes par kilo, en majorité sous forme organique et 0,15 gramme de méthymercure par kilo de poids sec dans les vases. Un phénomène de biomagnification amplifia le risque, puisque les concentrations de mercure dans le foie des poissons étaient 100000 fois plus fortes que la concentration dans l'eau de mer. Les concentrations maximales de mercure dans les coquillages et les poissons atteignirent respectivement $179 \mathrm{mg} / \mathrm{kg}$ de poids $\mathrm{sec}$ (norme OMS $2,5 \mathrm{mg} / \mathrm{kg}$ de poids sec) et $23 \mathrm{mg} / \mathrm{kg}$ de poids frais (norme OMS 0,5 $\mathrm{mg} / \mathrm{kg}$ de poids frais). Les concentrations de mercure dans les cheveux des malades pouvaient monter jusqu'à $705 \mu \mathrm{g} / \mathrm{kg}$ (pour une norme OMS de 10).

La symptomatologie observée chez les habitants de cette baie releva majoritairement de la neurotoxicité du méthylmercure et de son pouvoir cumulatif dans l'organisme. Elle s'est traduite par une restriction du champ visuel, des troubles de la sensibilité, des troubles du comportement avec ataxie cérébelleuse et incoordination des mouvements, altération de la parole et de l'audition, altération de la marche, tremblements et troubles mentaux légers .

Le lien entre l'épidémie et la présence de mercure étant établi, l'activité de la société a été arrêtée, la pêche a été interdite pendant plus de quarante ans. Des opérations gigantesques de dragage ont été menées pour retirer 1,5 million de $\mathrm{m}^{3}$ de sédiments. Les concentrations ont graduellement diminué. L'épidémie a causé au moins 48 morts, 158 invalides, incapables de subvenir seuls à leurs besoins, et 1742 victimes.

D'autres accidents collectifs ont également provoqué au Guatemala et surtout en Iraq près de 300 décès et 
plusieurs milliers d'hospitalisations dans les années 70 . Cette intoxication fut consécutive à l'alimentation du bétail et des volailles par des céréales traitées par un fongicide à base de méthylmercure en vue de les utiliser comme semences et de les conserver durant l'hiver. Le blé distribué était livré en sacs portant la mention $\mathrm{du}$ danger. Les paysans manquant d'aliments administrèrent les graines à leurs animaux. Ne constatant pas initialement de manifestations anormales, ils utilisèrent même ce blé pour la panification. Mais lorsque les animaux commencèrent à présenter des signes d'intoxication, les éleveurs s'empressèrent d'abattre et de vendre leurs bêtes, ce qui déclencha les intoxications humaines. On dût interdire la consommation de viande, mais aussi celle du poisson car, craignant des représailles, les paysans avaient déversé dans le Tigre, des sacs de céréales contaminées (34).

\section{Cas du bismuth}

Le bismuth est considéré comme l'un des éléments lourds le moins toxique. On l'appelle parfois de façon amusante l'élément « vert », sur le plan environnemental. Les sels de bismuth sont largement utilisés et ont trouvé diverses applications en pharmacie (traitement de l'ulcère), comme pigment en cosmétologie, dans l'industrie (pigments, alliages, céramiques, supraconducteurs) . Les sels les plus utilisés en pharmacie sont ou étaient le tartrate double de potassium et de bismuth, l'aluminate, le carbonate, le sous gallate, le nitrate basique et le salicylate.

Bien que la plupart des sels de bismuth soit très peu solubles dans l'eau à $\mathrm{pH}$ voisin de la neutralité, l'élément semble montrer une forte susceptibilité à la biométhylation. Le triméthylbismuth (TMBi), composé volatil, a été retrouvé dans les gaz issus de décharges municipales. La vitesse de transformation du $\mathrm{Bi}$ en TMBi dans les boues est environ 100 et même jusqu'à 4000 fois plus élevée que la transformation de As en composés volatils correspondants. Cette forte conversion du Bi pourrait être due à la méthylation de ce métal par la microflore ou à des facteurs extrinsèques comme la formation de complexes facilitant son absorption par les cellules microbiennes (35).

\section{L'encéphalopathie bismuthique}

L'intoxication par le bismuth et ses composés survient plus fréquemment lors d'un usage thérapeutique ou à la suite d'expositions professionnelles. Elle est responsable de déficiences rénales et de troubles mentaux. Ainsi 100 cas d'encéphalopathie dont certains mortels ont été rapportés en France et en Australie, après inges- tion de sous gallate et de sous nitrate de bismuth dans les années $70(36,37)$. Bien que l'étiologie de l'encéphalopathie bismuthique demeure encore obscure, quelques auteurs ont suggéré que la microflore de l'intestin pourrait être responsable de la conversion des sels de Bi en composés liposolubles, conduisant ainsi à une absorption plus forte par l'organisme humain ou bien seraient convertis en TMBi plus toxique qui serait responsable de l'encéphalopathie observée expérimentalement chez le chat et le chien .

Michalke et al.(35) ont montré récemment qu'une bactérie méthanogène commune dans les boues d'épuration, Methanobacterium formicium était capable de biométhyler le bismuth de différentes spécialités pharmaceutiques à base de ce métal (sous gallate, sous nitrate et aluminate). Les expérimentations in vitro suggèrent que la méthylation de $\mathrm{Bi}$ par cette bactérie est principalement catalysée par voie enzymatique faisant appel à la méthylcobalamine comme donneur de groupement méthyle. Les auteurs admettent que la flore méthanogénique de l'intestin est tout à fait capable de convertir ces médicaments en TMBi dont la haute stabilité en conditions anaérobiques serait responsable de l'accumulation de ce composé toxique. Reconnaissons que dans les années 70 on ne disposait pas des techniques d'analyse aussi précises et fiables que celles d'aujourd'hui et qu'il n'était donc possible que d'émettre des hypothèses quant à l'espèce toxique incriminée. Bien que l'étiologie de cette intoxication demeure encore du domaine hypothétique, la démonstration de cette équipe de chercheurs allemands apparaît fortement convaincante !

\section{Cas de l'étain}

Nous ne reviendrons pas sur les problèmes posés par les organostanniques dans l'environnement, notamment avec les composés tributylétains ; ils ont déjà été évoqués précédemment (38). Rappelons cependant que l'étain est un métal très anciennement connu mais ce n'est que dans la dernière partie du XIX ${ }^{\text {sme }}$ siècle que sa commercialisation a été mise au point et depuis cette époque l'étain et ses dérivés ont fait l'objet de nombreuses recherches qui ont permis de mettre en évidence plusieurs propriétés antiparasitaires ou antimicrobiennes débouchant sur des utilisations agricoles, vétérinaires et thérapeutiques.

\section{L'affaire du Stalinon®}

À la suite des travaux de Kolmer en 1931, qui avait montré l'action antistaphylococcique de certains composés organostanniques, le triiodure de méthylétain 
$\mathrm{CH}_{3} \mathrm{SnI}_{3}$ fut proposé pour le traitement de la furonculose. Quelques années plus tard, l'utilisation dans le même but du diiodure de diéthylétain $\left(\mathrm{C}_{2} \mathrm{H}_{5}\right)_{2} \mathrm{SnI}_{2}$ (STALINON®) provoqua un grand nombre d'intoxications dont plusieurs furent mortelles ( 102 morts sur 210 cas rapportés). Il semblerait que ce soit une ou plusieurs impuretés qui aient été responsables des effets toxiques. En effet, contrairement aux intentions, la composition du produit n'était pas uniforme et renfermait comme principale impureté un peu de triiodure d'éthylétain et environ $10 \%$ d'iodure de triéthylétain. Ce dernier composé s'est révélé environ dix fois plus toxique chez les mammifères que le dérivé diéthylé.

La symptomatologie de cette intoxication dramatique a été particulièrement bien décrite par Alajouanine et al (39). Dans les premiers jours qui suivent l'absorption, il apparaît des signes neurologiques avec céphalées, troubles sensoriels, vertiges, acouphènes. Puis s'installent brusquement, souvent à bas bruit, une encéphalopathie hyperthermique ; cette hyperthermie s'expliquant par le fait que le triéthylétain est reconnu comme l'un des plus puissants découplants de la phosphorylation oxydative mitochondriale. Des paraplégies d'installation brutale et très étendues ont souvent laissé de lourdes séquelles motrices. La mort des suites de l'intoxication orale par le Stalinon ${ }^{\circledR}$ est survenue dans $47 \%$ des cas.

\section{Brefs aspects analytiques}

Afin de pouvoir établir un lien de causalité direct entre les effets délétères observés et la responsabilité d'un agent toxique donné il faut pouvoir démontrer la présence dans les matrices biologiques ou environnementales, de cet agent ou espèce incriminé. Depuis longtemps on sait que le dosage global d'un élément métal ou métalloïde dans un milieu défini ne signifie plus rien. Nous avons remarqué dans ce qui a été écrit plus haut que ce sont parfois certains métabolites intermédiaires (As,Bi) ou des composés formés dans l'environnement $(\mathrm{Hg})$ ou enfin des impuretés de produits synthétiques utilisés dans un but thérapeutique $(\mathrm{Bi}, \mathrm{Sn})$ qui se sont révélés responsables de la toxicité observée. Ce sont donc ces formes chimiques (ionique ou moléculaire) d'un analyte qu'il faut rechercher, identifier et doser. Pour cela on fait appel aujourd'hui aux méthodes dites de spéciation qui associent à la séparation par chromatographie gazeuse (GC), liquide (LC) ou l'électrophorèse capillaire (EC), des techniques de détection hautement spécifiques comme le plasma à couplage inductif lié à la spectrométrie de masse (ICP-MS), la spectrométrie d'absorption atomique (AAS) ou enfin la spectrométrie de fluorescence atomique (AFS). $\mathrm{Au}$ cours de la dernière décennie, ces techniques ont été appliquées à diverses matrices environnementales (eau, sédiments) ou biologiques. Nous ne donnerons qu'un aperçu très général sur les moyens de résoudre aujourd'hui ce problème technique sans aborder les étapes initiales essentielles que constituent le prélèvement, le stockage et la préparation de l'échantillon à analyser.

Pour les échantillons gazeux, on procède au préalable à un piégeage à froid pour concentrer les analytes puis à une séparation par chromatographie gazeuse ; la séparation des espèces organométalliques s'effectue sur colonne remplie et la détection est réalisée par différentes méthodes : AAS, AFS, ICP-MS. On peut également séparer les organométalliques sur colonne capillaire suivie d'une détection par spectrométrie d'émission atomique (AES) ou ICP-MS. Les échantillons solides et liquides sont soumis à une extraction à l'aide de solvants appropriés puis concentrés après purification de l'extrait et analysés par les méthodes précitées.

L'HPLC présente de nombreux avantages par rapport à la GC pour les analyses de spéciation des métaux : les composés qui présentent un haut point d'ébullition peuvent être séparés sans modification chimique, par dérivation. La séparation est alors réalisée à des températures inférieures à celles utilisées en GC, ce qui rend ainsi l'HPLC préférable pour l'analyse des espèces thermosensibles. C'est aussi une méthode plus intéressante que la GC car la séparation est basée sur des interactions entre la phase stationnaire et la phase mobile et pas seulement avec la phase stationnaire comme en GC. L'HPLC est souvent utilisée dans les études monoélémentaires comme la spéciation de l'arsenic. Par exemple Kohlmeyer et al.(40) ont récemment publié une méthode permettant la séparation simultanée de 17 éspèces arsenicales organiques et minérales en une seule étape chromatographique. On a aussi décrit des méthodes HPLC pour la séparation et l'analyse du MMAs ${ }^{\mathrm{III}}$ et du DMAs ${ }^{\mathrm{III}}$ et autres composés arsénicaux présents dans l'environnement ou les milieux biologiques $(41,42,43)$. Harrington en 2000 a publié une revue sur la spéciation du mercure et des organomercuriels utilisant l'HPLC (44).

L'analyse des échantillons environnementaux requière des limites de détection basses ; c'est pourquoi la GC/ICP-MS est supérieure à l'HPLC/ICP-MS(1). En conséquence les échantillons liquides et solides sont souvent dérivés en espèces volatiles, par exemple grâce à la formation d'hydrures avec $\mathrm{NaBH} 4$ et analysés par GC/ICP-MS. Le tétraéthylborate est parfois utilisé pour la spéciation des métaux et métalloides dans les échantillons environnementaux. L'alkylation (éthylation, propylation en milieu aqueux ou butylation anhydre) a été préconisée pour la spéciation du mercure dans les 
échantillons biologiques. La spéciation de l'étain dans les matrices biologiques a été revue récemment par Anger (45). Chaque méthode de dérivation a ses avantages et ses inconvénients spécifiques et la spéciation comme la quantification des composés organométalliques dans les matrices complexes devrait être vérifiée par au moins deux techniques différentes.

\section{Conclusion}

La méthylation des métaux qu'elle soit naturelle ou voulue par l'homme n'est pas sans risque pour sa santé. Au travers de quelques exemples, nous avons voulu montrer que si les bactéries méthylent les métaux, c'est pour elles une forme de détoxication qui leur permet de diminuer la concentration cellulaire locale en ions métalliques toxiques dont elles se débarrassent par diffusion accrue. Le même phénomène a d'ailleurs été observé in vitro sur des lignées cellulaires que l'on peut rendre résistantes à différents métaux qu'elles expulsent grâce à des protéines de transport membranaires spécifiques. L'homme pour sa part ne semble pas s'adapter aussi facilement que les microorganismes et pour lui le vieil adage de Paracelse «Sola dosis fecit venenum » s'applique toujours. Lorsque l'homme perturbe l'équilibre environnemental, joue à l'apprenti sorcier ou élabore lui-même ces soi-disant produits de détoxication, il se trouve exposé à ces espèces organométalliques, par le biais de sa nourriture ou d'un traitement médical et subit de plein fouet les conséquences de son imprudence ou de son ignorance. En effet la plupart des composés organométalliques se révèlent comme des poisons neurotoxiques et génotoxiques. Il importe donc de surveiller leur présence dans l'environnement ou dans les milieux biologiques. Les méthodes modernes de spéciation alliant la séparation chromatographique à une détection spécifique et très sensible, permettent aujourd'hui de surveiller les niveaux d'exposition et devraient à l'avenir, souhaitons le, éviter de tels problèmes de santé.

\section{Références}

1. Dopp E., Hartmann L., Florea A.M., Rettenmeir A.W.,Hirner A.V. Environmental Distribution, Analysis and Toxicity of Organometal(oid) compounds. Crit. Rev. Toxicol. 2004, 34(3) : 301-33.

2. His W. Ueber das Stoffwechselproduct des Pyridins. Arch. Exp. Pathol. Pharmakol.1887,22 : 253-60.

3. Bentley R., Chasteen T.G. Microbial methylation of metalloids : Arsenic, Antimony and Bismuth. Microbiol. Mol. Biol. Rev. 2002,66(2) : 250-71.

4. Rosen B. P. Families of arsenic transporters. Trends Microbiol. 1999,7 : 207-12.
5. Challenger F., Higginbottom C.,Ellis L. The formation of organo-metalloïdal compounds by micro-organisms. Part.1 :Trimethylarsine and dimethylarsine. J. Chem. Soc., 1933 : 95-101.

6. Challenger F, Biological methylation. Adv. Enzymol. 1951, $12: 432-91$.

7. Thomas D.J., Waters S. B., Styblo M. Elucidating the pathway for arsenic methylation. Toxicol. Appl. Pharmacol. 2004, 198 : 319-26.

8. Cheng C.N., Focht D.D. Production of arsine, methylarsines in soil, in culture. Appl. Environ. Microbiol. 1979, 38 : 494-8.

9. Cullen W.R., Reimer J.R., Arsenic speciation in the environment. Chem. Rev. 1989, 89 : 713-64.

10. Laperche V., Bodénan F., Dictor M.C., Baranger Ph. Guide méthodologique de l'arsenic appliqué à la gestion des sites et sols pollués. 2003 BRGM/RP-52066 FR, 90 pp.

11. Tamaki S., Frankenberger Jr W.T. Environmental biochemistry of arsenic. Rev. Environ. Toxicol. 1992,124 : 79-110.

12. Styblo M.L., Drobna Z., Jaspers I., Lin S., Thomas D.J. The role of biomethylation in toxicity and carcinogenicity of Arsenic : a research update. Environ. Health Perspect $2002,110(5): 767-71$.

13. Hayakawa T., Kobayashi Y., Cui X., Hirano S. A new metabolic pathway of arsenite : arsenic-glutathione complexes are substrates for human arsenic methyltransferase Cyt 19. Arch. Toxicol. 2005,79(4) : 183-91.

14. Alauddin M ; Alauddin S.T., Bhattacharjee M., Sultana S., Chowdhury D., Bibi H., Rabbani G.H. Speciation of arsenic metabolite intermediates in human urine by ionexchange chromatography and flow injection hydride generation atomic absorption spectrometry. J. Environ. Sci. Health 2003,38,1 : 115-28.

15.Zakharian R.A., Wu Y., Bogdan G.M., Aposhian H.V. Enzymatic methylation of arsenic compounds. I. Assay, partial purification, and properties of arsenite methyltransferase and monomethylarsonic acid methyltransferase of rabbit liver. Chem. Res. Toxicol. 1995,8 : 1029-38.

16. Wildfang E., Zakharian R.A., Aposhian H.V. Enzymatic methylation of arsenic compounds. VI. Characterization of hamster liver arsenite and methylarsonic acid methyltransferase activities in vitro. Toxicol. Appl. Pharmacol. 1998,152: 366-75.

17. Styblo M., Del Razo L.M., LeCluyse E.L., Hamilton G. A., Wang C., Cullen W.R., Thomas D. J. Metabolism of arsenic in primary cultures of human and rat hepatocytes. Chem. Res. Toxicol. 1999,12 : 560-5.

18. Marafante E., Vahter M., Envall J. The role of the methylation in the detoxication of arsenate in the rabbit. Chem. Biol. Interact. 1985, $56: 225-38$.

19. Healy S.M., Wildfang E., Zakharian R.A., Aposhian H.V. Diversity of inorganic arsenite biotransformation. Biol. Trace Elem. 1999,68 : 249-66.

20. Vahter M., Mechanisms of arsenic biotransformations. Toxicology 2002,181-182 : 211-7.

21. Chiou H.Y., Hsueh Y.M., Hsieh L.L., Hsu Y.H., Hsieh F.I., Wei M.L., Chen H.C., Yang H.T., Leu L.C., Chu T.H., Chen-Wu C., Yang M.H., Chen J.C. Arsenic methylation capacity, body retention and null genotypes of glutathione S-transferases M1 and T1 among current arsenic-exposed residents in Taiwan. Mutat. Res 1997, 386 : 197-207. 
22. Hsueh Y.M., Huang Y.L., Huang C.C., Wu W.L., Chen H.M. , Yang M H., Lue L.C., Chen J.C. Urinary levels of inorganic and organic arsenic metabolites among residents in an arseniasis-hyperendemic aera in Taiwan. J. Toxicol. Environ. Health 1998, 54 : 431-44.

23. Concha G., Vogler G., Nermell B., Vahter M. Metabolism of inorganic arsenic in children with chronic high arsenic exposure in nothern Argentina. Environ. Health Perspect. 1998, 106(6) : 355-9.

24. Concha G., Vogler G., Lezcano D., Nermell B., Vahter M. Exposure to inorganic arsenic metabolites during early human development. Toxicol. Sci. 1998, 44(2) : 185-90.

25. Buchet J.P., Lauwerys R., Roels H. Urinary excretion of inorganic arsenic and its metabolites after repeated ingestion of sodium metaarsenite by volunteers. Int. Arch. Occup. Environ. Health 1981, 48 : 111-8.

26.Le X.C., Ma M., Lu X., Cullen W.R., Aposhian H.V., Zheng B. Determination of monomethylarsonous acid, a key arsenic methylation intermediate, in human urine. Environ. Health Perspect. 2000, 108(11) : 1015-8.

27. Styblo M., Del Razo L.M., Vega L., Germolec D.R., LeCluyse E.L., Hamilton G.A., Reed W., Wang C., Cullen W.R., Thomas D.J. Comparative toxicity of trivalent and pentavalent inorganic and methylated arsenicals in rat and human cells. Arch. Toxicol. 2000, 74 : 289-99.

28. Kitchin K.T. Recent advances in arsenic carcinogenesis : modes of action, animal model systems and methylated arsenic metabolites. Toxicol. Appl. Pharmacol 2001, 172 : 249-61.

28. Del Razo L.M., Garcia-Vargas G.G., Albores A., Gonsebatt M.E., Montero R., Ostrosky-Wegman P., Kelsh M., Cebrian M E. Altered profile of urinary arsenic metabolites in adults with chronic arsenicism. A pilot study. Arch. Toxicol. 1997, $71:$ 211-7.

29. Yu R.C., Hsu K. H., Chen J. C., Froines J.R. Arsenic methylation capacity and skin cancer. Cancer Epidemiol. Biomarkers Prev. 2000, 9 : 1259-62.

30. Mäki-Paakkanen J., Kurttio P., Paldy A., Pekkanen J. Association between the clastogenic effect in peripheral lymphocytes and human exposure to arsenic through drinking water. Environ. Mol. Mut. 1998, 32 : 301-13.

31. Goyer R.A. Toxic effects of metals. In Casarett and Doull's. Toxicology : The Basic Science of Poisons, $5^{\text {th }}$ Ed ; Klaassen C.D. Ed ; McGraw-Hill : New York,1996 : 696-8.

32. Boudène C. Rapport sur la toxicité des amalgames dentaires au mercure. Académie Nationale de Médecine, $1^{\text {er }}$ avril 2003 : 1-12. http://agmed.santé.gouv.fr/htm/10/dentaire/rptacmed.pfd.
33. Cumont G. La contamination des aliments par le mercure. Ann. Fals. Exp. Chim. 1984, 829 : 309-20.

34. Michalke K., Meyer J., Hirner A.V., Hensel R. Biomethylation of bismuth by the methanogen Methanobacterium formicium. Appl Organometal. Chem. 2002, $16: 221-7$.

35. Burge A., Rancurel G., Poisson M., Dechy H. Encéphalopathies myocloniques induites par les sels de bismuth. A propos de 6 cas. Nouv. Presse. Med 1974, 3 : 2315-20.

36. Burns R., Thomas D.W., Barron V.J. Reversible encephalopathy possibly associated with bismuth subgallate ingestion.Br. Med. J. 1974, $1: 220-3$.

37. Anger J.P. L'étain et les organo-étains dans l'environnement. Ann. Toxicol. Anal. 2001, 13 : 196-202.

38. Alajanouine T., Derobert L., Thieffry S. Etude clinique d'ensemble de 210 cas d'intoxication par les sels organiques de l'étain. Rev. Neurol 1958, 98(2) : 85-96.

39. Kolmeyer V., Kuballa J., Jantzen E. Simultaneous separation of 17 inorganic and organic arsenic compounds in marine biota by means of high-performance liquid chromatography/inductively coupled plasma mass spectrometry. Rapid Commun Mass Spectrom. 2002, 16 : 965-74.

40. Gailer J., Madden S., Cullen W.R., Denton M.B. The separation of dimethylarsinic acid, methylarsinous acid, methylarsonic acid, arsenate and dimethylarsinous acid on the Hamilton PRP-X100 anion-exchange column. Appl. Organomet. Chem. 1999, 13 : 837-48.

41. Gong Z., Lu X., Ma M., Watt. C., Le C. Arsenic speciation analysis. Talanta 2002, $58: 77-96$.

42. Hansen H.R., Raab A., Feldman J. New arsenosugar metabolite determined in urine by parallel use of HPLC-ICPMS and HPLC-ESI-MS. J. Anal. At. Spectrom. 2003, 18 : 474-9.

43. Zheng J, Hintelman H.Hyphenation of high performance liquid chromatography with sector field inductively coupled plasma mass spectrometry for the determination of ultra-trace level anionic and cationic arsenic compounds in freshwater fish. J. Anal. At. Spectrom.2004, 19 : 191-5.

44. Harrington C.F. The speciation of mercury and organomercury compounds by using high performance liquid chromatography. Trends Anal. Chem. 2000, 19 : 167-81.

45. Anger J.P. Tin in Meriam E ., Anke M., Inhat M., Stoeppler M. ed. Elements and their compounds in the Environment. 2ndEdition. Wiley-VCH Verlag $\mathrm{GmbH}$ \&Co,2004 : 1113-24. 